\title{
Risk-Based AED Placement - Singapore Case
}

\author{
Ivan Derevitskii ${ }^{1}$, Nikita Kogtikov ${ }^{3}$, Michael H. Lees $^{2}$, Wentong Cai ${ }^{3(凶)}$, \\ and Marcus E. H. Ong ${ }^{4}$ \\ 1 ITMO University, Saint-Petersburg, Russia \\ 2 University of Amsterdam, Amsterdam, The Netherlands \\ 3 School of Computer Science and Engineering, \\ Nanyang Technological University, Singapore 639798, Singapore \\ aswtcai@ntu.edu.sg \\ 4 Singapore General Hospital, Singapore, Singapore
}

\begin{abstract}
This paper presents a novel risk-based method for Automated External Defibrillator (AED) placement. In sudden cardiac events, availability of a nearby AED is crucial for the surviving of cardiac arrest patients. The common method uses historical Out-of-Hospital Cardiac Arrest (OHCA) data for AED placement optimization. But historical data often do not cover the entire area of investigation. The goal of this work is to develop an approach to improve the method based on historical data for AED placement. To this end, we have developed a riskbased method which generates artificial OHCAs based on a risk model. We compare our risk-based method with the one based on historical data using real Singapore OHCA occurrences from Pan-Asian Resuscitation Outcome Study (PAROS). Results show that to deploy a large number of AEDs the risk-based method outperforms the method purely using historical data on the testing dataset. This paper describes our risk-based AED placement method, discusses experimental results, and outlines future work.
\end{abstract}

Keywords: AED placement $\cdot$ Cardiac arrest $\cdot$ Optimization

\section{Introduction}

Cardiovascular disease accounts for near 18 million deaths a year worldwide, nearly $30 \%$ of all deaths [1]. Around $50 \%$ of these deaths are caused by sudden cardiac events and without intervention only $1 \%$ of people survive such an event. Automated External Defibrillators (AEDs) are one of the keyways to reduce mortality rates due to cardiac arrest. To impact survival rate, the time between the arrest and application of the AED is crucial. For this reason, governments worldwide have initiated AED deployment strategies in an effort to reduce deaths caused by sudden cardiac events. However, the current strategies are often driven by best practice, heuristics and guidelines (see [2]). 
More recently there have been efforts to attempt more analytical approaches, where historical data and projected cardiac events are used to optimize the deployment of AEDs. In these optimization approaches there are a number of key questions or challenges that need to be addressed: firstly, how to make the projection of future cardiac events; secondly, how to determine the suitability of the placement (i.e., the likelihood of an AED successfully preventing death); and finally a method or technique to optimize the deployment.

In this paper we propose a novel risk-based method and compare it with the existing method that uses purely historical data. Our method is based on an artificial cardiac arrest model that uses demography data and cardiac arrest odd ratio for different race groups. We study the case of Singapore, a small but densely populated country that has a highly varied demography and an ageing population [3]. In Singapore cardiac arrests are one of the most common causes of death, causing over 2,000 deaths annually [4]. In 2015, the Out-of-Hospital Cardiac Arrest (OHCA) survival rate in Singapore was 23.4\% [4]; whereas in other countries (e.g., the Netherlands) this can be as high as 40\% [5]. Through our novel optimization approach, we aim to improve survivability for the current population, but also to identify an AED deployment that can deal with future demographic changes for the country.

For our analysis we used data that capture all OHCAs in Singapore from 2010 to 2016, which contains a total of 11,861 occurrences. For the method based on historical data, we optimized the deployment using different amount of data. For all the approaches, we tested how the deployment would have performed for the OHCAs taking place in 2016. We say that the AED deployment successfully "covers" an OHCA if there is at least one AED within $100 \mathrm{~m}$ - a standard rule used by many other researchers (e.g., [6]). The results demonstrate that the riskbased approach provides better coverage than the method just using historical data especially when a large number of AEDs need to be deployed. Moreover, the artificial population approach can be used to generate arbitrary number of future cardiac arrests. This allows for scenario planning where population wide exogenous effects may increase OHCA rates (e.g., national trends in diet, national trends in exercise, etc.).

The remainder of this paper is organized as follows. Section 2 describes the existing service point optimization methods. Datasets used in the paper are presented in Sect.3. The research methodology and the proposed risk-based method are briefly described in Sect. 4 . Section 5 discusses optimization results. Finally, Sect. 6 concludes the paper with directions of future work.

\section{Related Works}

The area of AED placement optimization is already an active field of research, and it fits within a broader research area of service point optimization. In the following two subsections we provide an overview of the existing work in service point optimization as well as a comprehensive overview of the existing approaches for AED placement optimization. 


\subsection{Service Point Relocation Methods}

Optimization of service points is used in medicine [7-9], finance [10,11], as well as in the placement of commercial service points [12]. For spatial optimization, greedy algorithms [13], tabu-search [14], binary optimization [15], and genetic algorithms [16-18] are used. In [19], optimization of adding service points to an existing service network was proposed. However, access to a service point is often not round-the-clock and depends on time, therefore it is necessary to consider not only the spatial but also the temporal nature of service requests [20].

Often the nature of service requests depends on external data (for example, demographic data when optimizing commercial service points, or data on pedestrian and transport activity when optimizing the location of emergency medical facilities). Therefore, in this work, in addition to a method based on historical data (service requests), a method based on the demography data to determine OHCA subzone risk is proposed.

The complexity of the optimization problem may also depend on the characteristics of a particular locality and with it the features of the distribution of service requests [21]. Therefore, even the use of the existing methods is of interest for AED placement in Singapore.

\subsection{AED Relocation Works}

Chan et al. proposed a method based on historical data as well as a demographybased approach for AED placement in [19]. They used data of OHCA in Toronto. The dataset was divided into training and testing datasets. The training dataset was used to generate the input for the linear programming optimization and the testing dataset was used for evaluating optimization results. For the demographybased approach, people's daily activities were also considered during the optimization.

Dahan et al. proposed a method based on the historical OHCA data in [22]. They studied spatiotemporal optimization, which considers not only spatial, but also temporal availability of AEDs. Tsai et al. compared spatiotemporal approach and temporal only approach in terms of OHCA coverage in [23]. Sun et al. also studied spatiotemporal AED placement optimization with historical OHCA data in [20]. A cost-based optimization method for AED placement was proposed in [24], where cost effectiveness of the demography-based and the historical-data based method was compared. Instead of using the standard $100 \mathrm{~m}$ rule to determine the OHCA coverage, walking time was used in the fitness function for OHCA coverage estimation in [25].

The main disadvantage of the historical-data based method is the limited area covered by the dataset; whereas the demography-based method mentioned above does not consider cardiac arrest risk for different race groups in the population. Existing studies show that ethnicity is an important factor that affects OHCA risk. For example Malay race group have higher OHCA risk comparing to Chinese [26]. In this paper, we propose a risk-based method which considers race groups in estimating cardiac arrest risk and generates artificial OHCAs as 
the training dataset. A modified version of the optimization algorithm proposed in [19] is used in our work.

\section{Data}

Three datasets are used in this paper. The first dataset contains historical OHCAs, the second one describes the spatial demography of Singapore, and the third one provides the current (as of 6 May 2019) AED locations within Singapore. The historical OHCA dataset was obtained from Pan-Asian Resuscitation Outcome Study (PAROS). It covers all OHCA cases in Singapore from April 2010 to September 2016. This includes a total of 11,861 cases. Along with the location (given as location postcode) and the time at which symptom first occurred, associated with each OHCA there is basic demographic information about the patient such as age, ethnicity, and gender. Table 1 shows some details about this dataset, including cases per year as well as the division of cases among different race groups.

Table 1. OHCA dataset

\begin{tabular}{l|r|r|r|r|r|r|r}
\hline Year & 2010 & 2011 & 2012 & 2013 & 2014 & 2015 & 2016 \\
\hline Chinese & 742 & 893 & 984 & 1225 & 1349 & 1587 & 1253 \\
\hline Malay & 165 & 201 & 222 & 265 & 362 & 363 & 280 \\
\hline Indian & 99 & 185 & 146 & 161 & 209 & 277 & 200 \\
\hline Others & 75 & 98 & 88 & 85 & 117 & 145 & 85 \\
\hline All & 1081 & 1377 & 1440 & 1736 & 2037 & 2372 & 1818 \\
\hline
\end{tabular}

Table 2. Similarity between testing and training datasets

\begin{tabular}{l|l|l|l|l|l}
\hline $2010-2015$ & $2011-2015$ & $2012-2015$ & $2013-2015$ & $2014-2015$ & 2015 \\
\hline $48.86 \%$ & $45.89 \%$ & $40.58 \%$ & $35.1 \%$ & $28.14 \%$ & $18.32 \%$ \\
\hline
\end{tabular}

For the results reported in Sect. 5, to test the effectiveness of the method based on historical data we group historical data into six training datasets: OHCA occurrences in 2015-2015, 2014-2015, 2013-2015, 2012-2015, 2011-2015, and 2010-2015. These training datasets are used respectively by the optimization algorithm to obtain AED placements. To test optimization results, OHCA occurrences in 2016 are used. Table 2 shows similarity between the testing and training datasets based on postcodes. Note that similar to [20], OHCAs occurring in the healthcare facilities (e.g., nursing home) are excluded in both training and testing datasets. 
Table 3. Demography of town center subzone in Ang Mo Kio (2015)

\begin{tabular}{l|l|l|l}
\hline Chinese & Malay & Indian & Others \\
\hline 4260 & 210 & 360 & 190 \\
\hline
\end{tabular}

In order to generate potential future OHCA cases (and to make forecast), we use publicly available spatial demographic dataset from Singapore Data Mall (see link [27] for race-gender and [28] for age-gender data). This dataset divides Singapore into a total of 323 subzones [29] (see Fig. 1). Within each subzone the dataset provides statistics on the number of people and distributions of age, race, and gender. Table 3 illustrates an example of demography of town center subzone in Ang Mo Kio (a township in Singapore) in 2015.

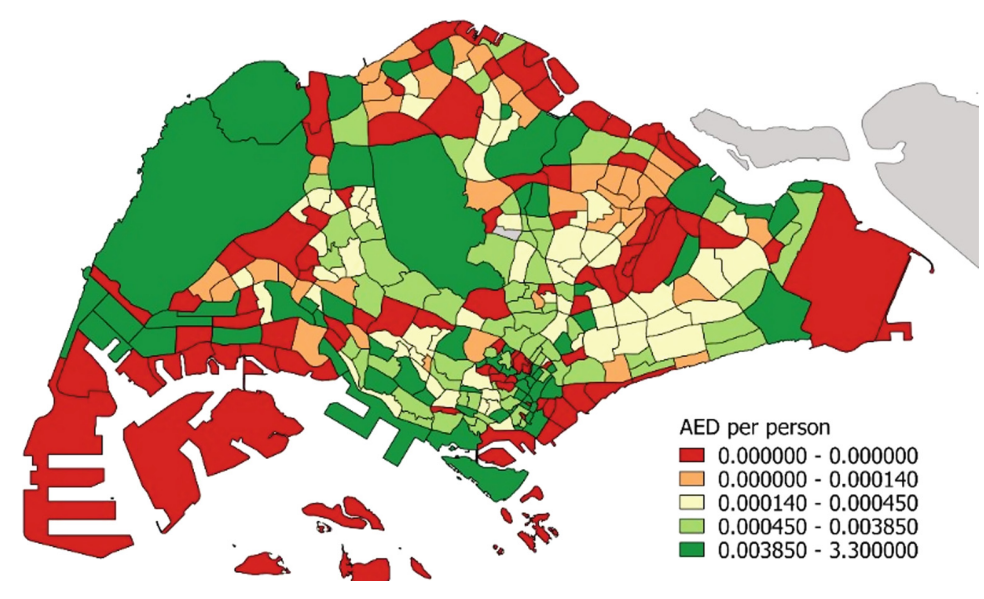

Fig. 1. A Map of Singapore divided into sub regions (each region shows the number of AEDs placed in that region per individual)

The third dataset includes the locations (longitude-latitude) of current 9,200 AEDs in Singapore (as of May 2019). Figure 1 shows the subzone boundaries as well as the AED density (AED per person) heatmap according to the dataset. It shows that the AED density varies across the entire Singapore island. However, the figure does not indicate the land use of each of the areas. For example, the large areas to the south-west (Jurong Island and Tuas) and to the east (Changi Airport) are non-residential areas. Within some other subzones there are also areas with very few residents.

There are a number of important caveats that we must mention regarding assumptions and inaccuracies about the data we use. Firstly, Singapore currently provides a number of mobile AEDs via the SMRT taxi company [30], the AEDs are placed within 1000 vehicles in the fleet. This accounts for an extra $12 \%$ of 
AEDs that are currently available and are not captured in our dataset. However, these AEDs were placed at the end of 2015 so their impact on the results should be minimal. Secondly, during our optimization procedure we assume that all building locations on the map are potential AED sites and that these sites are available for $24 \mathrm{~h}$. In reality, some locations may be inaccessible to the public, or only accessible during office hours. Building data were obtained via Open Street Map [31] and this dataset includes 141,821 locations of buildings in Singapore.

\section{Research Methodology}

In this paper we develop a risk-based method for AED placement, and to analyze its effectiveness we compare the result we obtain with alternative AED placement strategies. These include the current real-world AED placement and a traditional approach that simply uses historical occurrences of cardiac arrests to optimize the placement of AEDs. In this section we describe historical-data based and risk-based methods in detail, along with the underlying optimization technique and the evaluation criterion which assesses the effectiveness of a particular AED placement solution.

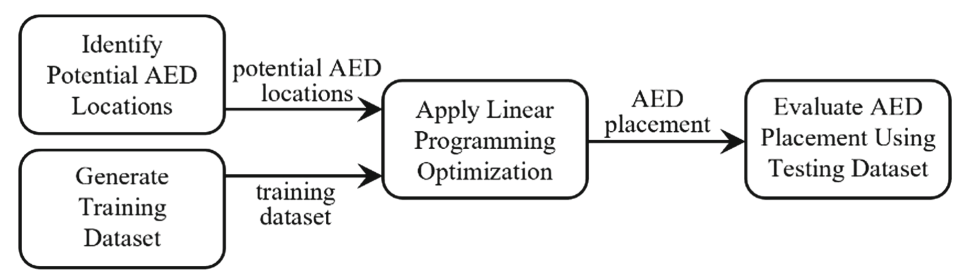

Fig. 2. Overview of research methodology

Figure 2 shows an overview of the research methodology. Initially the dataset regarding potential AED locations is created. Potential locations (buildings) are identified using Open Street Map. This means that the AED optimization is considered at the resolution of a building, and the exact placement of the AED within the building is not explicitly modelled. All methods use the same set of potential locations. The training dataset used by the optimization algorithm differs in the case of the historical-data based approach and the risk-based method. In the historical-data based approach, as explained in Sect. 3 we group OHCA occurrences in 2010-2015 into six training datasets; whereas in the risk-based method artificial OHCAs are generated based on the demographics of the population in 2015.

The potential location data and the training data are then combined by creating a weighted adjacency matrix, where the weights of the edges are calculated as the straight-line distances from each potential AED location to each OHCA location in the training data. This matrix is used as the input data in the linear programming optimization algorithm (described below and based on [19]). 
Finally, an AED placement generated is evaluated by testing how many of the 2016 OHCA cases would be covered by the placement.

\subsection{Optimization Approach and Evaluation Criterion}

The input to the optimization algorithm takes the form of an adjacency matrix as described above. In this paper we use a modified version of the linear programming algorithm in [19] for finding optimal location of AEDs. This is a binary optimization problem (also known as the Maximal Covering Location Problem [15]), defined as follows:

$$
\operatorname{maximize} \sum_{j=1}^{J} x_{j} ; \text { subject to } \sum_{i=1}^{I} y_{i}=N \text { and } x_{j} \leq \sum_{i=1}^{I} a_{i j} y_{i}, \forall j=1, \ldots, J
$$

where $x_{j}$ is a binary parameter indicating whether cardiac arrest $j$ is covered or not; $y_{i}$ is a binary decision variable indicating whether an AED is placed in location $i$ or not; and $a_{i j}$ is binary parameter indicating whether cardiac arrest $j$ is coverable by location $i$ (i.e., within $100 \mathrm{~m}$ ). $N$ is the number of locations in which AEDs are placed. $I$ is the number of potential locations where AEDs can be placed. $J$ is the number of cardiac arrests in the training dataset.

Each of the methods will produce a placement of AEDs, that is a mapping of $N$ AEDs to potential locations. From this placement an assessment is made to see what fraction of OHCAs recorded in 2016 would have been covered. As mentioned above, in this paper we use a simple measure of coverage, that is, an OHCA is considered covered by the placement if it is within $100 \mathrm{~m}$ from the nearest AED. This does not include the time it takes for an individual to locate the AED, or any vertical distance that must be covered.

\subsection{Historical-Data Based Method}

The historical-data based method tries to optimize the AED placement under the assumption that previous OHCA occurrences are a good predictor for future OHCA cases. We have data from a six-year period 2010-2016. In this approach, we use different combinations of historical data as a predictor for future OHCAs. In particular, we use 2016 as our testing dataset and see if the data from 20152015, 2014-2015, 2013-2015, 2012-2015, 2011-2015, and 2010-2015 are good predictors for the OHCAs in 2016. The effectiveness of this approach is determined by the demographic stability in Singapore. If the occurrence of OHCAs are clustered and stable over a period of years then this approach should be effective on some time scale.

\subsection{Risk-Based Method}

The risk-based method tries to capture various factors that might predict future OHCA cases, including population density and distribution of population in 
terms of ethnicity. Using the current population data, we can identify high risk subzones of the Singapore island. From this a set of potential or artificial OHCAs can be generated, which will be used as the training dataset to optimize the placement of AEDs.

Singapore is a multi-cultural city with most of the country's inhabitants coming from four major ethnic groups: Chinese (74.3\%), Malay (13.4\%), Indians $(9 \%)$ and others $(3.2 \%)$, this distribution has remained remarkably stable since Singapore's independence (more than 50 years) [32]. As in other countries [33] the risk of heart disease and cardiac arrest does vary by race. Using the OHCA data we can derive an associated risk of OHCA for a specific race group, $r_{\text {race }}$, as follows:

$$
r_{\text {race }}=\frac{\sum_{\text {year }=2010}^{2015} n_{\text {race }}^{\text {year }}}{\sum_{\text {year }=2010}^{2015} p_{\text {race }}^{\text {year }}} \times \frac{\sum_{\text {year }=2010}^{2015} p_{\text {Chinese }}^{\text {year }}}{\sum_{\text {year }=2010}^{2015} n_{\text {Chinese }}^{\text {year }}}
$$

where race $\in\{$ Chinese, Malay, Indian,Others $\}$; $n_{\text {race }}^{\text {year }}$ is the number OHCA cases for a specific race in a specific year; $p_{\text {race }}^{\text {year }}$ is the number of people in Singapore for a specific race in a specific year. Note that race group risk is estimated using historical OHCA occurrences in 2010-2015 and that the value is normalized with the OHCA risk of the Chinese race group. The calculated risk values for various race groups are shown in Table 4.

Table 4. Risks for different races

\begin{tabular}{l|l|l|l}
\hline Chinese & Malay & Indian & Others \\
\hline 1.000 & 1.181 & 1.635 & 4.833 \\
\hline
\end{tabular}

Given an associated risk for each race group, we then calculate an associated risk for each subzone of Singapore. To do this we use the data about the total population size and race distributions in each of Singapore's subzones in 2015 (see Sect. 3 for detail). Subzone OHCA risk, $R_{s}$, is calculated as follows:

$$
R_{s}=\frac{\sum_{i \in\{\text { Chinese,Indian,Malay }, \text { Others }\}} r_{i} \times \text { dem }_{i, s}}{\text { dem }_{s}}
$$

where $r_{i}$ is the OHCA risk for race group $i$; dem ${ }_{i, s}$ is the number of people in race group $i$ who live in subzone $s$ in 2015; and $d_{e} m_{s}$ is the total number of people living in subzone $s$ in 2015.

Based on subzone OHCA risks, a zonal risk map can be generated. As shown in Fig. 3, we use a simple algorithm to generate a set of potential/artificial OHCAs. To generate a location for a potential OHCA, a subzone is first chosen based on subzone OHCA risks. The higher the subzone OHCA risk is, the more often a subzone will be chosen. After that a random location (building) will be selected within the subzone for the location of OHCA. This procedure terminates when the required 


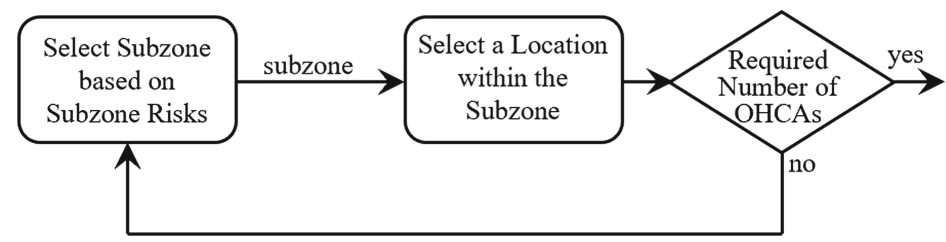

Fig. 3. Artificial OHCA generation

number of artificial OHCAs are generated. These artificial OHCAs then serve as training dataset to the optimization algorithm (see Fig. 2). With this method we can in principle generate any number of OHCAs. The number of artificial OHCAs is currently set as a parameter in our experiments.

\section{AED Optimization Result}

In this section we demonstrate the results of different optimization methods presented in Sect. 4 and compare their performance against the current deployment of AEDs. Recall that in all scenarios the performance of the deployment is based on the fraction of OHCAs occurring in 2016 (a total of 1,818 cases) that would be covered by at least one AED (that is, there is at least one AED existing within $100 \mathrm{~m})$.

\subsection{Historical-Data Based Method}

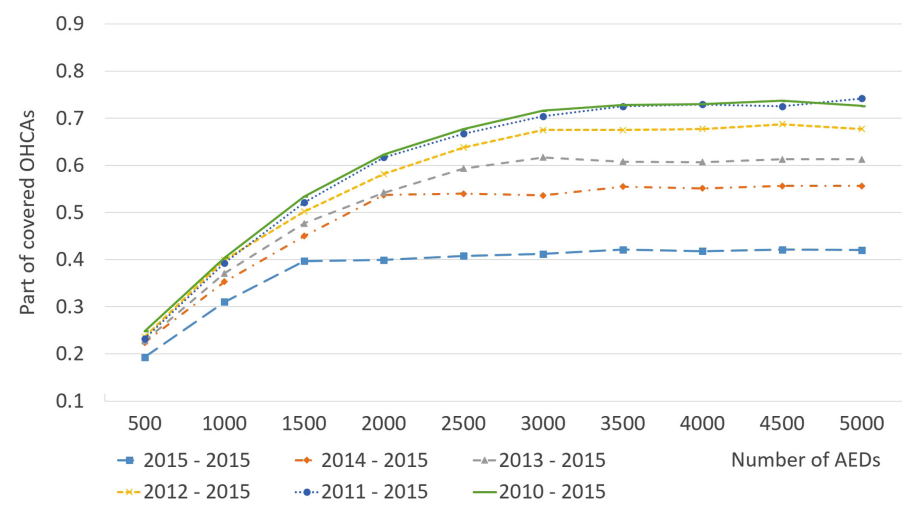

Fig. 4. Results of historical-data based method for different training datasets

Figure 4 shows the result of historical-data based method using 6 different training datasets (from 1-year data 2015-2015 to 6-year dataset 2010-2015). It clearly shows the performance depends on the training dataset. Table 1 presents the 
number of OHCAs for each year, which gives a clear indication of the size of each training dataset. Due to the increase of similarity between training and testing datasets (see Table 2), for a given number of AEDs to be deployed, there is an increasing improvement on the OHCA coverage when more data are added into the training dataset. However, the improvement diminishes as more historical data are added to the training dataset since the increase of similarity becomes smaller (see Table 2). In principle however, more data do not necessarily imply better performance. This depends on the dynamics of the population and how uniform the aging process is across the country. Due to the housing policy it is likely that the most at risk subzones may move as the population of particular housing developments age.

All scenarios shown in Fig. 4 have similar performance when a small number of AEDs are deployed because the optimization algorithm tends to put AEDs first in the areas that have frequent OHCA occurrences over the years. When a large number of AEDs are deployed, the larger datasets obviously work better because they cover larger area and have higher similarity with the training dataset (as showed in Table 2). Note that all the curves become flat after the number of AEDs increases to a certain level. This is because even the largest 6-year training dataset does not cover all areas in Singapore and its similarity with the testing dataset is only $48.86 \%$. The figure also shows that as the amount of training data increases, the limit of maximal coverage will also increase (from $43 \%$ for the 1-year data to $74 \%$ for 6 -year data).

\section{$5.2 \quad$ Risk-Based Method}

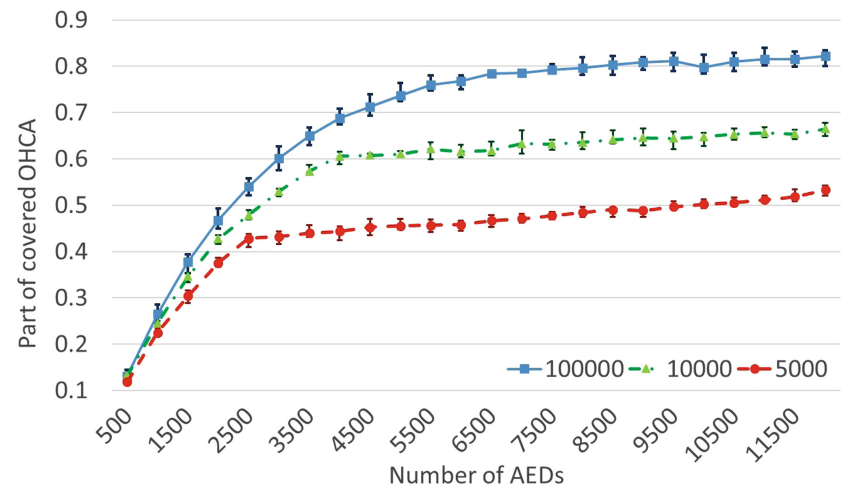

Fig. 5. Results of risk-based method for different training dataset

Figure 5 shows the results of the risk-based method. Recall that the risk-based method uses artificial OHCAs generated from subzone risks as training dataset for the optimization algorithm (see Sect.4). In our experiments, the number of generated artificial OHCAs in the training dataset varies from 5,000, 10,000, to 
100,000. Obviously, for a given number AEDs to be deployed, better coverage is achieved with larger training dataset. Interestingly, we see a saturation effect similar to the historical-data based method for the training dataset with 5,000 and 10,000 artificial OHCAs. The advantage of using the risk-based method is that we can generate the training dataset with arbitrarily large number of artificial OHCAs. Note that the largest training dataset used in the historicaldata based method (i.e., the 6-year dataset) contains only 10,043 OHCA cases.

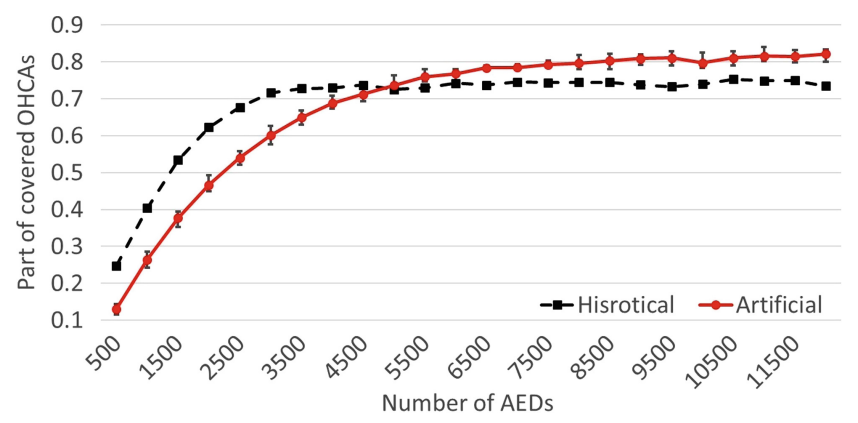

Fig. 6. Comparison between historical-data based and risk-based methods

Figure 6 compares the best results obtained using the historical-data based and risk-based methods. When the number of AEDs to be deployed is small, historical-data based method performs better than the risk-based method. However, when a large number of AEDs needs to be deployed, the risk-based method would be a better choice. In order words, the training dataset used in the riskbased method is able to provide more meaningful information to guide the deployment of large number of AEDs. The performance crossover happens at around 6,500 AEDs. For a reference, there are currently 9,203 AEDs deployed in Singapore (as of 6 May 2019).

\section{Conclusion}

We studied the problem of optimizing AED placement in Singapore. Both the historical-data based and the risk-based methods were used in our investigation. OHCA occurrences from 2010 to 2015 in Singapore were used as the training datasets in the historical-data based method. In the risk-based method, historical OHCAs and demography data were used to derive subzone OHCA risks in Singapore, which in turn were used to generate potential or artificial OHCAs in the training dataset. The performance of both methods was evaluated using OHCA occurrences in 2016.

The results demonstrate a number of interesting findings. Firstly, irrespective of the training datasets used in the historical-data based method, the first 2,000 AEDs placed have an equally effective coverage of OHCAs. Secondly, for all the 
training dataset used in the historical-data based method, the OHCA coverage does not increase further after the number of AEDs reaches a certain level. Finally, for a small number of AEDs, the historical-data based method gives a better OHCA coverage than the risk-based method. However, when a large number of AEDs needs to be deployed, the risk-based method would be a better choice.

As for our future work, to combine the two methods, the risk model can be used to generate artificial OHCAs to complement historical data. Most of the existing work determines OHCA coverage either in terms of distance or time, to better define OHCA coverage we will be developing a probabilistic model using historical OHCA data and AED location data. Countries around the world are dealing with ageing populations. The dynamics of this ageing can introduce very unique risks, which may be geographically correlated. We are also planning to investigate how these changing risk maps for cardiac arrests may impact the placement of AEDs.

Acknowledgments. This work was supported by the National Research Foundation (NRF) of Singapore, GovTech, under its Virtual Singapore Program Grant No. NRF2017VSG-AT3DCM001-031. Ivan Derevitskii is also financially supported by The Russian Science Foundation, Agreement №17-71-30029 with co-financing from Bank Saint Petersburg.

\section{References}

1. World Health Organization. https://www.who.int/news-room/fact-sheets/detail/ cardiovascular-diseases-(cvds). Accessed Apr 2020

2. Resuscitation Council (UK). https://www.resus.org.uk/publications/a-guide-to-a. Accessed Apr 2020

3. Department of Statistics Singapore. https://www.singstat.gov.sg/-/media/files/ visualising_data/infographics/population/population-trends2019.pdf. Accessed Apr 2020

4. Singapore Heart Foundation. https://www.myheart.org.sg/programmes/save-alife-initiative/. Accessed Apr 2020

5. Boyce, L.W., et al.: High survival rate of $43 \%$ in out-of-hospital cardiac arrest patients in an optimised chain of survival. Netherlands Heart J. 23(1), 20-25 (2015)

6. Chan, T.C.Y., Demirtas, D., Kwon, R.H.: Optimizing the deployment of public access defibrillators. Manage. Sci. 62(12), 3617-3635 (2016)

7. Brotcorne, L., Laporte, G., Semet, F.: Ambulance location and relocation models. Eur. J. Oper. Res. 147, 451-463 (2003)

8. Baray, J.Ô., Cliquet, G.: Optimizing locations through a maximum covering/pmedian hierarchical model: maternity hospitals in France. J. Bus. Res. 66(1), 127$132(2013)$

9. Li, X., Zhao, Z., Zhu, X., Wyatt, T.: Covering models and optimization techniques for emergency response facility location and planning: a review. Math. Methods Oper. Res. 74(3), 291-310 (2011)

10. Allahi, S., Mobin, M., Vafadarnikjoo, A., Salmon, C.: An integrated AHP-GISMCLP method to locate bank branches. In: Industrial and Systems Engineering Research Conference (ISERC), Nashville, Tennessee, USA, June 2015 
11. Miliotis, P., Dimopoulou, M., Giannikos, I.: A hierarchical location model for locating bank branches in a competitive environment. Int. Trans. Oper. Res. 9(5), 549565 (2002)

12. Wu, S., Kuang, H., Lo, S.-M.: Modeling shopping center location choice: shopper preference-based competitive location model. J. Urban Plan. Dev. 145(1), 04018047 (2018)

13. Tong, D., Murray, A., Xiao, N.: Heuristics in spatial analysis: a genetic algorithm for coverage maximization. Ann. Assoc. Am. Geogr. 99(4), 698-711 (2009)

14. Basar, A., Kabak, Ö., Topcu, Y.I.: A tabu search algorithm for multi-period bank branch location problem: a case study in a Turkish bank. Scientia Iranica 26, 3728-3746 (2018)

15. Church, R., ReVelle, C.: The maximal covering location problem. Papers of the Regional Science Association, vol. 32, no. 1, pp. 101-118 (1974)

16. Beasley, J.E., Chu, P.C.: A genetic algorithm for the set covering problem. Eur. J. Oper. Res. 94(2), 392-404 (1996)

17. Aickelin, U.: An indirect genetic algorithm for set covering problems. J. Oper. Res. Soc. 53(10), 1118-1126 (2002)

18. Solar, M., Parada, V., Urrutia, R.: A parallel genetic algorithm to solve the setcovering problem. Comput. Oper. Res. 29, 1221-1235 (2002)

19. Chan, T.C.Y., et al.: Identifying locations for public access defibrillators using mathematical optimization. Circulation 127(17), 1801-1809 (2013)

20. Sun, C.L.F., Demirtas, D., Brooks, S.C., Morrison, L.J., Chan, T.C.: Overcoming spatial and temporal barriers to public access defibrillators via optimization. J. Am. Coll. Cardiol. 68(8), 83-845 (2016)

21. Hansen, C.M., et al.: Automated external defibrillators inaccessible to more than half of nearby cardiac arrests in public locations during evening, nighttime, and weekends. Circulation 128(20), 2224-2231 (2013)

22. Dahan, B., et al.: Optimization of automated external defibrillator deployment outdoors: an evidence-based approach. Resuscitation 108, 68-74 (2016)

23. Tsai, Y.S., Ko, P.C.-I., Huang, C.-Y., Wen, T.-H.: Optimizing locations for the installation of automated external defibrillators (AEDs) in urban public streets through the use of spatial and temporal weighting schemes. Aplli. Geogr. 35(1-2), 394-404 (2012)

24. Tierney, N.J., et al.: Novel relocation methods for automatic external defibrillator improve out-of-hospital cardiac arrest coverage under limited resources. Resuscitation 125, 83-89 (2018)

25. Bonnet, B., Dessavre, D.G., Kraus, K., Ramirez-Marquez, J.E.: Optimal placement of public-access AEDs in urban environments. Comput. Ind. Eng. 90, 269-280 (2015)

26. Rakun, A., et al.: Ethnic and neighborhood socioeconomic differences in incidence and survival from out of hospital cardiac arrest in Singapore. Prehosp. Emer. Care 23(5), 619-630 (2019)

27. Singapore government data. https://data.gov.sg/dataset/resident-population-byplanning-area-subzone-ethnic-group-and-sex-2015. Accessed Apr 2020

28. Singapore government data. data.gov.sg/dataset/resident-population-by-planningarea-subzone-age-group-and-sex-2015. Accessed Apr 2020

29. Singapore subzones master plan. https://data.gov.sg/dataset/master-plan-2014subzone-boundary-web. Accessed Apr 2020

30. HeartSine. https://heartsine.com/2015/12/singapore-taxi-drivers-will-nowprotect-public-with-on-board-defibrillators/ 
31. OpenStreetMap. https://www.openstreetmap.org/. Accessed Apr 2020

32. Teo, T.-A.: Civic Multiculturalism in Singapore - Revisiting Citizenship. Rights and Recognition. Palgrave, London (2019)

33. Becker, B., et al.: Racial differences in the incidence of cardiac arrest and subsequent survival. N. Engl. J. Med. 329(9), 600-606 (1993) 